\title{
CONSTRUCTION OF FIXED POINTS FOR ASYMPTOTICALLY NONEXPANSIVE MAPPINGS
}

\author{
GREGORY B. PASSTY
}

\begin{abstract}
In uniformly convex Banach spaces with Fréchet differentiable norms (e.g. $L^{p}, 1<p<\infty$ ), fixed points for asymptotically nonexpansive mappings are constructed as weak limits of iterates of the mappings themselves or of related mappings.
\end{abstract}

Let $E$ be a uniformly convex Banach space, and let $C$ be a closed convex subset of $E$. A mapping $U$ of $C$ into itself is said to be asymptotically nonexpansive (Goebel and Kirk [6]) if $\left\|U^{n} x-U^{n} y\right\| \leqslant k_{n}\|x-y\|$ for all $x$ and $y$ in $C$, with $\lim _{n} k_{n}=1$. It was proved in [6] that if $C$ is further assumed to be bounded, then an asymptotically nonexpansive self-map of $C$ has a fixed point. We show here that if $E$ has a Fréchet differentiable norm, and if $U$ is, for example, weakly continuous, then fixed points of $U$ can be obtained by iterating $U$ starting at a point of asymptotic regularity.

Theorem 1 extends theorems of Feathers and Dotson [5] and of Bose [2] which were obtained in uniformly convex spaces with weakly continuous duality maps. The basic tool in both of these papers was Opial's Lemma [7]. Because this lemma does not carry over to $L^{p}, p \neq 2$, new techniques are needed for this more general case. These were provided by Baillon [1] and simplified by Bruck [4] when the norm is Fréchet differentiable.

We will present Theorem 1 in a slightly more general form, and then discuss applications to asymptotically nonexpansive mappings and to a conjecture of $\mathrm{H}$. Schaefer [8].

First we extend the definition of [6] to sequences of maps which are not necessarily powers of a given map.

Definition 1 . The sequence $\left\{T_{n}\right\}_{n=1}^{\infty}$ of self-maps of $C$ is asymptotically nonexpansive if $\left\|T_{n} x-T_{n} y\right\| \leqslant k_{n}\|x-y\|$ for all $x, y$ in $C$ with $\lim _{n} k_{n}=1$.

Denote the set of fixed points of $T$ by $F(T)$, strong convergence by $\rightarrow$, and weak convergence by $\rightarrow$. We may now state

THEOREM 1. Let $E$ be uniformly convex with a Fréchet differentiable norm, and $C a$ closed convex subset of $E$. Let $F$ be a subset of $C$ and $S=\left\{T_{n}\right\}_{n=1}^{\infty}$ an asymptotically nonexpansive sequence of self-maps of $C$ such that (a) $F \subset \cap_{n=1}^{\infty} F\left(T_{n}\right)$. Assume also that there exists $x_{0}$ in $C$ for which

(b) $T_{n_{i}} x_{0} \underset{i}{\rightarrow} z$ implies $z \in F$, and

Received by the editors April 24, 1981.

1980 Mathematics Subject Classification. Primary 47H10, Secondary 47H09. 
(c) $T_{n} T_{m} x_{0}-T_{n} x_{0} \rightarrow 0$ as $n \rightarrow \infty$ for all (fixed) $m$.

Then either (i) $F=\varnothing$ and $\left\|T_{n} x_{0}\right\| \rightarrow+\infty$ or (ii) $F \neq \varnothing$ and $T_{n} x_{0} \rightarrow$ an element of $F$.

Note that hypothesis (c) may be interpreted as asymptotic regularity of $S$ at $x_{0}$. In order to prove Theorem 1, we proceed using several lemmas.

LEMMA 1. Let $x_{0}$ and $y_{0}$ be any two elements of $C$ for which hypothesis (c) holds. Then $\lim _{n}\left\|T_{n} x_{0}-T_{n} y_{0}\right\|$ exists.

Proof. By the triangle inequality and the Lipschitz property of $T_{n}$,

$$
\begin{aligned}
\left\|T_{n} x_{0}-T_{n} y_{0}\right\| & \leqslant\left\|T_{n} x_{0}-T_{n} T_{m} x_{0}\right\|+\left\|T_{n} T_{m} x_{0}-T_{n} T_{m} y_{0}\right\|+\left\|T_{n} T_{m} y_{0}-T_{n} y_{0}\right\| \\
& \leqslant\left\|T_{n} x_{0}-T_{n} T_{m} x_{0}\right\|+k_{n}\left\|T_{m} x_{0}-T_{m} y_{0}\right\|+\left\|T_{n} T_{m} y_{0}-T_{n} y_{0}\right\| .
\end{aligned}
$$

Fixing $m$ and letting $n \rightarrow \infty$, applying (c), and then letting $m \rightarrow \infty$, we see

$$
\lim \sup _{n}\left\|T_{n} x_{0}-T_{n} y_{0}\right\| \leqslant \lim _{m} \inf \left\|T_{m} x_{0}-T_{m} y_{0}\right\| \text {. Q.E.D. }
$$

Corollary 1. For each $f \in F, \lim _{n}\left\|T_{n} x_{0}-f\right\|$ exists.

Proof. By (a), $T_{n} f=f$ for all $n$. In addition, $x_{0}$ (by assumption) and $f$ satisfy (c). Q.E.D.

A very important tool is a result proved by Bruck, which we state here as

LEMMA 2 [4]. Let $E$ be a uniformly convex Banach space and let $K$ be a nonempty closed bounded convex subset of $E$. Then there exists a strictly increasing, continuous convex function $\gamma: \mathbf{R}^{+} \rightarrow \mathbf{R}^{+}$with $\gamma(0)=0$ such that every nonexpansive mapping $U$ : $K \rightarrow E$ satisfies

$$
\gamma(\|r U x+(1-r) U y-U(r x+(1-r) y)\|) \leqslant\|x-y\|-\|U x-U y\|
$$

for all $x, y$ in $K$ and $0 \leqslant r \leqslant 1$.

More suitable to our purposes is a variant of Lemma 2 for mappings which are not necessarily nonexpansive.

COROLlary 2. Let $E, K$, and consequently $\gamma$ be as in Lemma 2, and let $T: K \rightarrow E$ be Lipschitz with Lipschitz constant $k$. Then

$$
\|r T x+(1-r) T y-T(r x+(1-r) y)\| \leqslant k \gamma^{-1}\left(\|x-y\|-k^{-1}\|T x-T y\|\right)
$$

for all $x$ and $y$ in $K$ and $0 \leqslant r \leqslant 1$.

We now begin the proof of Theorem 1, following lines developed in [4].

Proof of Theorem 1 (Beginning). Suppose some subsequence $\left\{T_{n_{i}} x_{0}\right\}$ is bounded. Since $E$ is reflexive, a further subsequence must converge weakly to an element $z \in E$ which, by (b), is in $F$. Thus $F=\varnothing$ implies $\left\|T_{n} x_{0}\right\| \rightarrow \infty$.

If, on the other hand, $F \neq \varnothing$, then there is some $f_{0} \in F$ and, by Corollary 1, $\left\{\left\|T_{n} x_{0}-f_{0}\right\|\right\}$ is bounded, say, by $R$. Let $C_{1}=\left\{x \in C:\left\|x-f_{0}\right\|<R\right\}$. Then $C_{1}$ is closed, convex, bounded, and nonempty. Furthermore, $T_{n} x_{0} \in C_{1}$ for all $n$. We take $C_{1}$ to be the set $K$ in Corollary 2 . 
We abstract the next section of the proof as

LEMMA 3. Under the hypotheses of Theorem $1, \lim _{n}\left\|r T_{n} x_{0}+(1-r) f_{1}-f_{2}\right\|$ exists for all $f_{1}, f_{2} \in F$ and for all $0 \leqslant r \leqslant 1$.

Proof.

$$
\begin{aligned}
\| r T_{n} x_{0} & +(1-r) f_{1}-f_{2}\|\leqslant r\| T_{n} x_{0}-T_{n} T_{m} x_{0}\|+\| r T_{n} T_{m} x_{0}+(1-r) f_{1}-f_{2} \| \\
\leqslant & r\left\|T_{n} x_{0}-T_{n} T_{m} x_{0}\right\|+\left\|r T_{n} T_{m} x_{0}+(1-r) T_{n} f_{1}-T_{n}\left(r T_{m} x_{0}+(1-r) f_{1}\right)\right\| \\
& +\left\|T_{n}\left(r T_{m} x_{0}+(1-r) f_{1}\right)-T_{n} f_{2}\right\| \\
\leqslant & r\left\|T_{n} x_{0}-T_{n} T_{m} x_{0}\right\|+k_{n} \gamma^{-1}\left(\left\|T_{m} x_{0}-f_{1}\right\|-k_{n}^{-1}\left\|T_{n} T_{m} x_{0}-f_{1}\right\|\right) \\
& +k_{n}\left\|r T_{m} x_{0}+(1-r) f_{1}-f_{2}\right\| \\
\leqslant & r\left\|T_{n} x_{0}-T_{n} T_{m} x_{0}\right\| \\
& +k_{n} \gamma^{-1}\left(\left\|T_{m} x_{0}-f_{1}\right\|-k_{n}^{-1}\left\|T_{n} x_{0}-f_{1}\right\|+k_{n}^{-1}\left\|T_{n} x_{0}-T_{n} T_{m} x_{0}\right\|\right) \\
& +k_{n}\left\|r T_{m} x_{0}+(1-r) f_{1}-f_{2}\right\| .
\end{aligned}
$$

Here we have used the triangle inequality, hypothesis (a), the Lipschitz property of $T_{n}$, Corollary 2 , the triangle inequality again, and the fact that $\gamma^{-1}$ is also an increasing function. Fix $m$ and let $n \rightarrow \infty$ :

$$
\begin{gathered}
\lim _{n} \sup _{n}\left\|r T_{n} x_{0}+(1-r) f_{1}-f_{2}\right\| \leqslant 0+\gamma^{-1}\left(\left\|T_{m} x_{0}-f_{1}\right\|-\lim _{n}\left\|T_{n} x_{0}-f_{1}\right\|\right) \\
+\left\|r T_{m} x_{0}+(1-r) f_{1}-f_{2}\right\| .
\end{gathered}
$$

Now letting $m \rightarrow \infty$,

$\lim _{n} \sup \left\|r T_{n} x_{0}+(1-r) f_{1}-f_{2}\right\| \leqslant \lim _{m} \inf \left\|r T_{m} x_{0}+(1-r) f_{1}-f_{2}\right\|$. Q.E.D.

Proof of Theorem 1 (Conclusion). Let $f_{1}, f_{2} \in F$. Since $E$ has a Fréchet differentiable norm, we may take $J(u)$ to be the Fréchet derivative of $\frac{1}{2}\|\cdot\|^{2}$ at $u$. Then there exists an increasing function $\lambda: \mathbf{R}^{+} \rightarrow \mathbf{R}^{+}$such that $t^{-1} \lambda(t) \rightarrow 0$ as $t \rightarrow 0^{+}$and

$$
\begin{aligned}
\frac{1}{2}\left\|f_{1}-f_{2}\right\|^{2}+(J( & \left.\left.f_{1}-f_{2}\right), h\right) \leqslant \frac{1}{2}\left\|f_{1}-f_{2}+h\right\|^{2} \\
& <\frac{1}{2}\left\|f_{1}-f_{2}\right\|^{2}+\left(J\left(f_{1}-f_{2}\right), h\right)+\lambda(\|h\|)
\end{aligned}
$$

for all $h \in E$. Take $h=r\left(T_{n} x_{0}-f_{1}\right)$. Then

$$
\begin{aligned}
\frac{1}{2}\left\|f_{1}-f_{2}\right\|^{2} & +r\left(J\left(f_{1}-f_{2}\right), T_{n} x_{0}-f_{1}\right) \leqslant \frac{1}{2}\left\|r T_{n} x_{0}+(1-r) f_{1}-f_{2}\right\|^{2} \\
& \leqslant \frac{1}{2}\left\|f_{1}-f_{2}\right\|^{2}+r\left(J\left(f_{1}-f_{2}\right), T_{n} x_{0}-f_{1}\right)+\lambda\left(r\left\|T_{n} x_{0}-f_{1}\right\|\right) .
\end{aligned}
$$

Letting $n \rightarrow \infty$ and using Lemma 3, we obtain

$$
\begin{aligned}
\limsup _{n} r\left(J\left(f_{1}-f_{2}\right), T_{n} x_{0}-f_{1}\right) & \leqslant \frac{1}{2} \lim _{n}\left\|r T_{n} x_{0}+(1-r) f_{1}-f_{2}\right\|^{2}-\frac{1}{2}\left\|f_{1}-f_{2}\right\|^{2} \\
& \leqslant \lim _{n} \inf r\left(J\left(f_{1}-f_{2}\right), T_{n} x_{0}-f_{\mathrm{k}}\right)+\lambda(r M),
\end{aligned}
$$


where $\left\|T_{n} x_{0}-f_{1}\right\| \leqslant M$ for all $n$. Dividing by $r$ and letting $r \rightarrow 0^{+}$shows $\lim _{n}\left(J\left(f_{1}-f_{2}\right), T_{n} x_{0}\right)$ exists for all $f_{1}, f_{2} \in F$. Thus if $z_{1}$ and $z_{2}$ are two weak subsequential limits of $\left\{T_{n} x_{0}\right\},\left(J\left(f_{1}-f_{2}\right), z_{1}-z_{2}\right)=0$. By (b), $z_{1}$ and $z_{2}$ are in $F$; thus we may take $f_{i}=z_{i}$ for $i=1,2$, finding that $0=\left(J\left(z_{1}-z_{2}\right), z_{1}-z_{2}\right)=$ $\left\|z_{1}-z_{2}\right\|^{2}$. (We have used $(J u, u)=\|u\|^{2}=\|J u\|^{2}$ for all $u \in E[3$, p. 97].) Since all weak subsequential limits of the bounded sequence $\left\{T_{n} x_{0}\right\}$ are thus equal, $\left\{T_{n} x_{0}\right\}$ must converge weakly to an element of $F$. Q.E.D.

We now present a consequence of Theorem 1 for weakly continuous mappings.

Corollary 3. Let $C$ be closed, convex, and bounded, and let $U: C \rightarrow C$ be weakly continuous and asymptotically nonexpansive. If $U$ is asymptotically regular at $x_{0} \in C$ (i.e., if $U^{n+1} x_{0}-U^{n} x_{0} \rightarrow 0$ ), then $U^{n} x_{0}$ converges weakly to a fixed point of $U$.

Proof. In Theorem 1, we take $F=F(U)$, and $S=\left\{U^{n}\right\}_{n=1}^{\infty}$. It is obvious that (a) holds, and (c) holds by assumption. To see that (b) holds, note that $U^{n_{j}} x_{0} \rightarrow z$ and weak continuity imply that $U^{n_{j}+1} x_{0} \rightarrow U z$. On the other hand, asymptotic regularity implies that $\left\{U^{n_{j}+1} x_{0}\right\}$ and $\left\{U^{n_{3}} x_{0}\right\}$ must have the same limit, namely $z$. Thus $U z=z . F \neq \varnothing$ by [6], so $\left\{U^{n} x_{0}\right\}$ must converge weakly to an element of $F$. Q.E.D.

Theorem 1 may also be used to make statements about nonexpansive mappings.

THEOREM 2. Let $E$ be uniformly convex with a Fréchet differentiable norm, let $C$ be a bounded closed convex subset of $E$, and let $U$ be a nonexpansive self-map of $C$.

Set $U_{\lambda}=\lambda U+(1-\lambda) I$ for fixed $0<\lambda<1$. Then for any $x \in C,\left\{U_{\lambda}^{n} x\right\}$ converges weakly to a fixed point of $U$.

RemarK. This theorem shows that Schaefer's conjecture [8] holds in a class of uniformly convex spaces which includes $L^{p}, 1<p<\infty$.

Proof of Theorem 2. We apply Theorem 1 with $F=F(U)$ and $S=\left\{U_{\lambda}^{n}\right\}_{n=1}^{\infty}$. $F(U)=F\left(U_{\lambda}\right)$ for all $0<\lambda<1$, so (a) holds. By the Browder-Kirk-Göhde Theorem, $U$ has a fixed point in $C . U_{\lambda}$ is thus asymptotically regular [8, Lemma 2] establishing (c) with $x_{0}$ any element of $C$. In order to verify (b), note that,by asymptotic regularity, $\left(I-U_{\lambda}\right) U_{\lambda}^{n} x \rightarrow 0$. Since $U_{\lambda}^{n} x \rightarrow z$ (by assumption) and since $\left(I-U_{\lambda}\right)$ is demiclosed [3, Theorem 8.4], we see that $0=\left(I-U_{\lambda}\right) z$; i.e. $z=U z$. Since $F$ is nonempty, $\left\{U_{\lambda}^{n} x\right\}$ must converge weakly to a fixed point of $U$. Q.E.D.

ADDED IN PROOF. The author has learned that Theorem 2 is a special case of a result of S. Reich (J. Math. Anal. Appl. 67 (1979), 274-276, Theorem 2).

\section{REFERENCES}

1. J. B. Baillon, Thèse, Université de Paris.

2. S. C. Bose, Weak convergence to the fixed point of an asymptotically nonexpansive map, Proc. Amer. Math. Soc. 68 (1978), 305-308.

3. F. E. Browder, Nonlinear operators and nonlinear equations of evolution in Banach spaces, Proc. Sympos. Pure Math., vol. 18, Part 2, Amer. Math. Soc., Providence, R. I., 1976.

4. R. E. Bruck, A simple proof of the Mean Ergodic Theorem for nonlinear contractions in Banach spaces, Israel J. Math. 32 (1979), 107-116. 
5. G. Feathers and W. G. Dotson, Jr., A nonlinear theorem of ergodic type. II, Proc. Amer. Math. Soc. 73 (1979), 37-39.

6. K. Goebel and W. A. Kirk, A fixed point theorem for asymptotically nonexpansive mappings, Proc. Amer. Math. Soc. 35 (1972), 171-174.

7. Z. Opial, Weak convergence of the sequence of successive approximations for nonexpansive mappings, Bull. Amer. Math. Soc. 73 (1967), 591-597.

8. H. Schaefer, Über die Methode sukzessiver Approximationen, Jber. Deutsch. Math.-Verein. 59 (1957), 131-140.

School of Mathematics, Georgia Institute of Technology, Atlanta, Georgin 30332 